International Journal of Modern Physics A

(C) World Scientific Publishing Company

\title{
Experiments for Neutrinoless Double-Beta Decay
}

\author{
Steven R. Elliott \\ Physics Division, Los Alamos National Laboratory \\ MS H803, P-23, Los Alamos, NM, 87545, USA
}

Received (Day Month Year)

Revised (Day Month Year)

\begin{abstract}
The recent neutrino oscillation experimental results indicate that at least one neutrino has a mass greater than $50 \mathrm{meV}$. The next generation of double-beta decay experiments will very likely have a sensitivity to an effective Majorana neutrino mass below this target. Therefore this is a very exciting time for this field of research as even null results from these experiments have the potential to elucidate the nature of the neutrino.
\end{abstract}

Keywords: Double-beta decay, Neutrino mass.

\section{Introduction}

Double-beta decay $(\beta \beta)$ is a nuclear process where the nucleus increases in charge by 2 while emitting 2 electrons. Two-neutrino double-beta decay $(\beta \beta(2 \nu))$ is an allowed second order weak process that also emits two neutrinos. Zero-neutrino double-beta decay $(\beta \beta(0 \nu))$ can only occur if neutrinos are massive particles that are self conjugate (i.e. massive Majorana particles).

The recent experimental results from atmospheric 12134 , solar 167789 , and reactor 101112 neutrino sources indicate neutrinos have mass and that they mix. In particular, at least one neutrino must have a mass greater than about $50 \mathrm{meV}$. This very exciting result has greatly renewed the interest in $\beta \beta(0 \nu)$ because the next generation experiments aim to reach this target. In fact of all the experimental techniques to search for neutrino mass, only $\beta \beta(0 \nu)$ experiments can reach an absolute mass value near the $50-\mathrm{meV}$ mass scale indicated by the oscillation experiments. Furthermore, only $\beta \beta(0 \nu)$ has the potential to determine whether the neutrino is its own anti-particle. This exciting situation and the present experimental and theoretical status has been described in a recent review 13 and the many references therein.

Although any observation of $\beta \beta(0 \nu)$ has always been an exciting possibility, in the not-to-recent past, limits on the Majorana mass from the non-observation of $\beta \beta(0 \nu)$ could be interpreted in two distinct ways. Perhaps the neutrino mass was just smaller than the limit. Or, since $\beta \beta(0 \nu)$ is only sensitive to Majorana neutrinos, it could be that the neutrino was a Dirac particle that has a mass greater than the 
limit. Now, however, there is a known minimum mass scale for the neutrino. A null result below this mass scale could eliminate presently-possible mass spectra from having a Majorana component. Therefore such a result would be a non-trivial constraint on neutrino properties.

The oscillation results convincingly indicate two mass-difference scales: One at $\delta m_{\text {atm }}^{2} \sim(50 \mathrm{meV})^{2}$ at approximately maximal mixing and one at $\delta m_{\text {sol }}^{2} \sim(7$ $\mathrm{meV})^{2}$ at a mixing angle near 30 degrees. These results indicate that neutrinos do have mass but do not give an indication of the absolute neutrino mass scale or its CP nature. Furthermore, we have little data on the hierarchy of the neutrino masses. That is we do not know if $\nu_{e}$ is predominately composed of the lightest mass eigenstate or not. $\beta \beta(0 \nu)$ can provide data to address these neutrino properties, but because of possible non-trivial Majorana phases in the mixing matrix, we will also require input from kinematic neutrino mass experiments such as Katrin 14 . Therefore, all three types of experiments, oscillation, $\beta \beta(0 \nu)$, and kinematic, are required to completely understand the neutrino. Note that indications of a third mass-difference scale near $1 \mathrm{eV}^{2}$ from the LSND experiment 15 are as yet unconfirmed. Although I have chosen to ignore that result in this simple discussion, the large mass scale would only add to the interest in $\beta \beta$ experiments.

The decay rate $(\Gamma)$ of double beta decay is proportional to the square of the effective Majorana neutrino mass $\left(\left\langle m_{\beta \beta}\right\rangle\right)$, an easily calculable phase space factor $(G)$, and a difficult-to-calculate nuclear matrix element $\left(M_{0 \nu}\right)$;

$$
\Gamma=G M_{0 \nu}\left\langle m_{\beta \beta}\right\rangle^{2} .
$$

The value for $\left\langle m_{\beta \beta}\right\rangle$ in turn, depends on the values of the individual neutrino mass eigenstates $\left(m_{i}\right)$, the mixing matrix elements $\left(U_{e i}\right)$ and the Majorana phases $\left(\alpha_{i}\right)$;

$$
\left\langle m_{\beta \beta}\right\rangle^{2}=\left.\left.\left|\sum_{i}^{N}\right| U_{e i}\right|^{2} e^{\alpha_{i}} m_{i}\right|^{2} .
$$

In the case of $\mathrm{CP}$ conservation $e^{\alpha_{i}}= \pm 1$. If we take $U_{e 3}=0$, the atmospheric mixing to be maximal, and the solar mixing angle to be about 30 degrees $\left(U_{e 1}^{2}=\right.$ $\left.0.25, U_{e 2}^{2}=0.75\right)$, one can simplify this equation for $\left\langle m_{\beta \beta}\right\rangle$ and get a feeling as to the sensitivity that $\beta \beta(0 \nu)$ has to the absolute mass scale.

$$
\left\langle m_{\beta \beta}\right\rangle \sim\left|U_{e 1}\right|^{2} m_{1} \pm\left|U_{e 2}\right|^{2} \sqrt{m_{1}^{2}+\delta m_{12}^{2}} .
$$

For Majorana neutrinos in the normal hierarchy, one finds $\left\langle m_{\beta \beta}\right\rangle$ values greater than several $\mathrm{meV}$ and in the inverse hierarchy one finds values greater than several tens of meV even when the smallest mass eigenstate is near zero. Simply stated, $\sqrt{\delta m_{\text {atm }}^{2}}$ is a very enticing target for $\beta \beta(0 \nu)$ experiments and the recent excitement in $\beta \beta(0 \nu)$ is that the next generation of experiments will be sensitive to $\left\langle m_{\beta \beta}\right\rangle$ near this target. 


\section{Backgrounds to Double-Beta Decay}

To observe $\beta \beta(0 \nu)$, one must reduce backgrounds so that an ultra-low-rate peak can emerge from a continuum of other processes. To reach the desired 50-meV goal, significant background improvements are needed. The backgrounds that currently afflict $\beta \beta(0 \nu)$ experiments can be classified into 3 groups: $\beta \beta(2 \nu)$, natural or manmade radioactivities, and cosmogenicly produced radioactivities.

\subsection{Two-Neutrino Double-Beta Decay as a Background}

Unlike $\beta \beta(0 \nu)$, the two electrons share the available energy with two neutrinos in the $\beta \beta(2 \nu)$ process. Thus their sum energy spectrum is a distribution up to the endpoint. (See Fig. 1.) This spectrum is very steeply falling and, in principle, the region of interest for $\beta \beta(0 \nu)$ should be free of such events. However, the finite resolution of any detector can result in $\beta \beta(2 \nu)$ events polluting the $\beta \beta(0 \nu)$ region. An approximate expression 13 for the $\left\langle m_{\beta \beta}\right\rangle$ sensitivity limit due to this background can be written

$$
\left\langle m_{\beta \beta}\right\rangle^{2} \sim \frac{7 Q \delta^{6}}{m_{e}} \frac{G_{2 \nu}}{G_{0 \nu}} \frac{\left|M_{2 \nu}\right|^{2}}{\left|M_{0 \nu}\right|^{2}}
$$

where $Q$ is the $\mathrm{Q}$-value, $\delta$ is the width of the region of interest divided by $Q, m_{e}$ is the electron mass, $G_{2 \nu}$ is the $\beta \beta(2 \nu)$ phase space factor, $G_{0 \nu}$ is the $\beta \beta(0 \nu)$ phase space factor, $\left|M_{2 \nu}\right|$ is the $\beta \beta(2 \nu)$ matrix element and $\left|M_{0 \nu}\right|$ is the $\beta \beta(0 \nu)$ matrix element.

Although this equation does not include the improvement one might get from exploiting other kinematic measurements such as the opening angle or the individual electron energies, it does give an estimate of the resolution requirements. This equation is plotted for a few isotopes in Fig. 2 and one sees that a resolution better a few percent will suffice for the 50-meV goal. However, if other backgrounds are present and create a continuum through the region of interest, better resolution improves the experimental sensitivity.

\subsection{Natural and Man-made Radioactivity}

Many $\beta \beta$ experiments also serve as dark matter searches. Those searches look for the low-energy recoils resulting from elastic scattering of Weakly Interacting Massive Particles (WIMPS). The potential background for those searches is more varied than for $\beta \beta(0 \nu)$. Because the $\beta \beta(0 \nu)$ endpoint is typically a few $\mathrm{MeV}$, many natural radioactivities simply contribute too little energy to pollute that region of interest.

The most important naturally occurring isotopes that are potential backgrounds for $\beta \beta(0 \nu)$ are ${ }^{208} \mathrm{Tl}$ and ${ }^{214} \mathrm{Bi}$. These have large $\mathrm{Q}$-values and can pollute the region of interest of almost all $\beta \beta$ isotopes. They are members of the natural Th and $\mathrm{U}$ decay chains and thus common in the environment. Furthermore, they are daughters of the gaseous $\mathrm{Rn}$ isotopes, which are very mobile. The Th and $\mathrm{U}$ half-lives, $\left(\approx 10^{10}\right.$ $\mathrm{y}$ ), are much shorter than the required $10^{26}$ to $10^{27}$-y sensitivity for the $50-\mathrm{meV}$ 


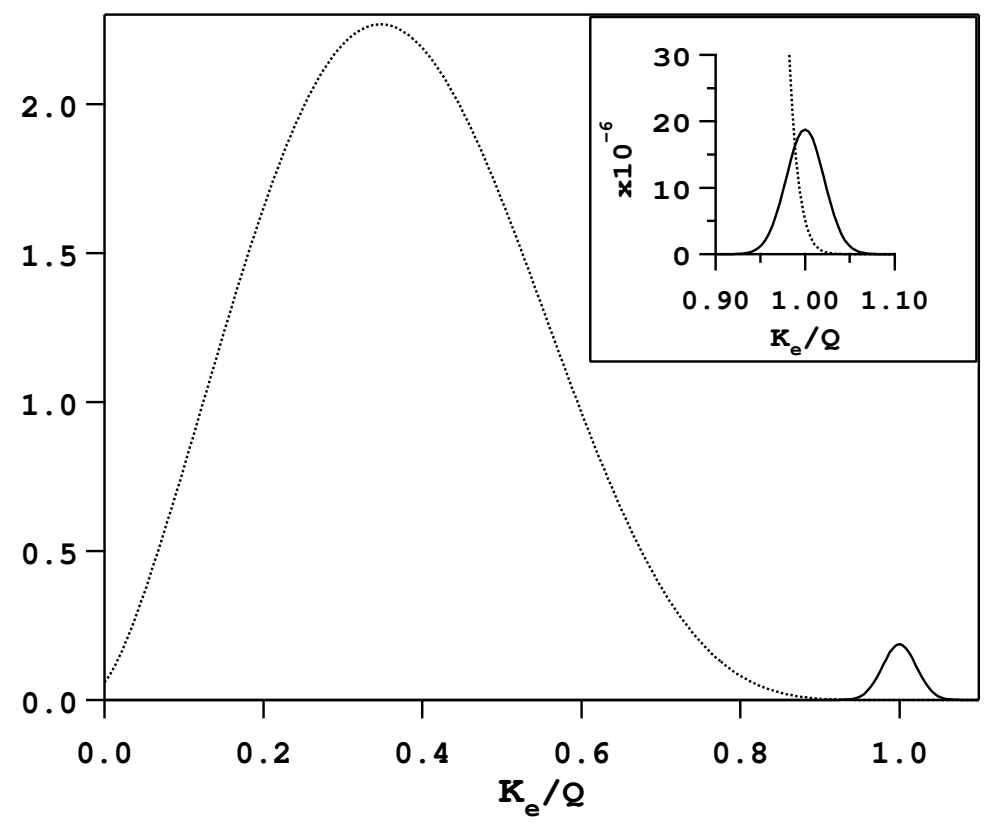

Fig. 1. Illustration of the spectra of the sum of the electron kinetic energies $\mathrm{K}_{e}$ ( $\mathrm{Q}$ is the endpoint) for the $\beta \beta(2 \nu)$ normalized to 1 (dotted curve) and $\beta \beta(0 \nu)$ decays (solid curve). The $\beta \beta(0 \nu)$ spectrum is normalized to $10^{-2}\left(10^{-6}\right.$ in the figure inset). All spectra are convolved with an energy resolution of $5 \%$, representative of several experiments. However, some experiments, notably Ge diodes and Te bolometers, have a much better energy resolution $(\approx 0.2 \%)$.

goal. Therefore even a tiny amount of these activities are a significant problem. Over the past 60 years, experimentalists have made great progress in identifying materials that are very low in Th and U. By building their experiments from this limited palate of materials, these activities have been greatly reduced. Improved purification techniques have also helped eliminate these backgrounds.

Radon is a special problem because its a gas that emanates from $\mathrm{U}$ and $\mathrm{Th}$ containing compounds and diffuses through many materials also. Experimenters must ensure that the detector volume is kept free of Rn. In many cases a careful flushing of the atmosphere near the inner volume with boil-off gas from liquid nitrogen sufficiently reduces the Rn. At $\mathrm{LN}$ temperatures, $\mathrm{Rn}$ is frozen out and therefore the boil-off gas is mostly free of Rn.

There are techniques to tag $\mathrm{Tl}$ and Bi background events based either on the kinematics of the decay processes or on delayed coincidence timing of the progenitors and daughter members of the natural decay chains. Although there has been great success in reducing backgrounds in this way, all these techniques have some 


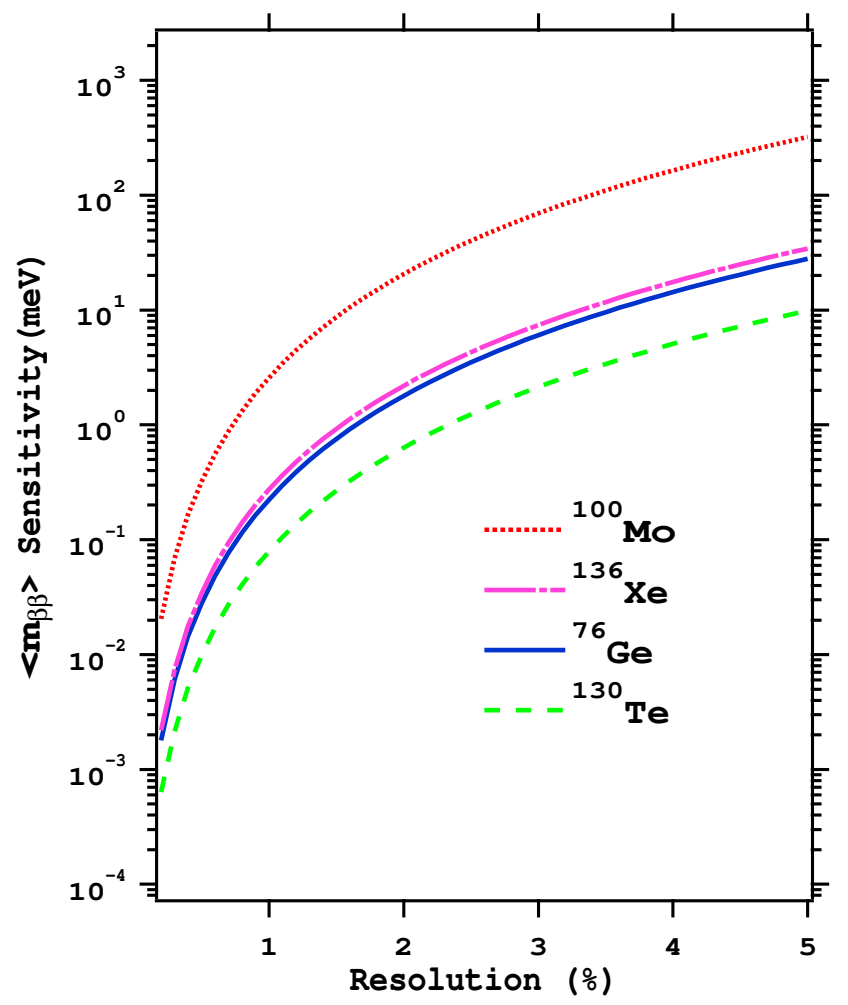

Fig. 2. The limit on the mass sensitivity as a function of sum energy resolution for a few isotopes.

inefficiency. Therefore it is necessary to minimize these activities. The future proposals will make great efforts to reduce the amount of $\mathrm{Tl}$ and $\mathrm{Bi}$ present even if they rely on such tagging techniques.

Many isotopes not normally found in nature (e.g. ${ }^{239,240} \mathrm{Pu},{ }^{137} \mathrm{Cs},{ }^{90} \mathrm{Sr},{ }^{42} \mathrm{Ar}$, and ${ }^{85} \mathrm{Kr}$ are produced artificially by human activities such as nuclear-weapon testing, nuclear accidents, reactor ventings, etc. Therefore it is necessary for experimenters to consider such exotic possibilities when designing an experiment.

\subsection{Cosmogenic Radioactivity}

Cosmic rays react with a detector and produce signals. Because the cosmic ray flux is so high on the surface of the Earth, $\beta \beta$ experiments are conducted underground. Any prompt events can be eliminated by going to a deep location and incorporating an anti-coincidence shield. But in addition to prompt interactions, cosmic rays can produce delayed radioactivity via many nuclear reactions. In particular, while detector materials or the source resides on the surface of the Earth, they are exposed 
to a significant fast $(>10 \mathrm{MeV})$ neutron flux. These fast neutrons can produce large $\Delta \mathrm{A}$ transitions in nuclei that result in radioactive nuclides.

Below ground the fast neutron flux is proportional to the cosmic-ray muon flux, so going deeper reduces it. For most cases, a few hundred meters will suffice to eliminate the in situ production and only the residual activity left over from the time spent on the surface will be present. The most famous example of this effect is that of ${ }^{68} \mathrm{Ge}$ in Ge detectors. Even though the experiments used Ge enriched in ${ }^{76} \mathrm{Ge},{ }^{68} \mathrm{Ge}$ was produced in the crystals through the high-threshold reaction, ${ }^{76} \mathrm{Ge}(\mathrm{n}, 9 \mathrm{n}){ }^{68} \mathrm{Ge}$. In using enriched Ge with little ${ }^{70,72,73,74} \mathrm{Ge}$, experimenters had thought that the ${ }^{68} \mathrm{Ge}$ problem would not be present because the required reaction on ${ }^{76} \mathrm{Ge}$ had such a large $\Delta \mathrm{A}$. Although it was significantly decreased, it remained a source of background that will require additional efforts to minimize.

For future experiments that will require sensitivities near 1 event/year in the region of interest in a 1-ton sample, the cosmogenic background possibilities are varied. Because the signal rate is very low in a large target, rare processes must be considered as potential backgrounds. For example ${ }^{92} \mathrm{Mo}(\mathrm{n}, 2 \mathrm{n}){ }^{91} \mathrm{Mo}$ would need to be carefully considered for any ${ }^{100} \mathrm{Mo}$ experiment using natural Mo. For a ${ }^{136} \mathrm{Xe}$ experiment trying to identify the ${ }^{136} \mathrm{Ba}$ daughter, the two-step process ${ }^{136} \mathrm{Xe}(\mu, \mathrm{p}) \mathrm{X}$ and ${ }^{136} \mathrm{Xe}(\mathrm{p}, \mathrm{n}){ }^{136} \mathrm{Cs}$ produces the 13.1 -day Cs isotope. ${ }^{136} \mathrm{Cs}$ decays via a $2.55-\mathrm{MeV}$ $\beta$ to ${ }^{136} \mathrm{Ba}$. Although it is unlikely that the energies of the $\beta$ and the coincident $\gamma \mathrm{s}$ would sum to the region of interest, it points out how rare (and sometimes bizarre) processes must be considered.

\section{An Ideal Experiment}

The first direct measurement of $\beta \beta(2 \nu)$ used a time projection chamber 16 . This was a fairly large apparatus $\left(\approx \mathrm{m}^{3}\right)$ for a modest amount of source $(13 \mathrm{~g})$ and therefore, it is doubtful that this type of arrangement will represent the best of the next generation of $\beta \beta(0 \nu)$ experiments. This design doesn't scale easily to very large source mass with very low backgrounds. It is interesting to try to enumerate the features that an ideal $\beta \beta(0 \nu)$ experiment would posses. It would have the following characteristics:

- The detector mass must be large enough to reach our $50-\mathrm{meV}$ goal $(\approx 1$ ton of isotope).

- The $\beta \beta(0 \nu)$ source must be extremely low in radioactive contamination.

- The proposal must be based on a demonstrated technology for the detection of $\beta \beta$.

- Although the use of natural isotope will be less costly, the enrichment process provides a good level of purification and also results in a (usually) much smaller volume detector.

- A small detector volume minimizes internal backgrounds, which scale with the detector volume. It also minimizes external backgrounds by minimizing the shield volume for a given stopping power. This is most easily accomplished by an ap- 
paratus whose source is also the detector. Alternatively, a very large source may have some advantage due to self shielding.

- Good energy resolution is required to prevent the tail of the $\beta \beta(2 \nu)$ spectrum extending into the $\beta \beta(0 \nu)$ region of interest.

- Ease of operation is required because these experiments usually operate in remote locations.

- A large $\mathrm{Q}$ value results in a fast $\beta \beta(0 \nu)$ rate and also places the region of interest above many potential backgrounds.

- A relatively slow $\beta \beta(2 \nu)$ rate also helps control this background.

- Identifying the daughter in coincidence with the $\beta \beta$ decay energy would eliminate most potential backgrounds except $\beta \beta(2 \nu)$.

- Event reconstruction, providing kinematic data such as opening angle and individual electron energy, can aid in the elimination of backgrounds. This data might also help elucidate the physics if a statistical sample of $\beta \beta(0 \nu)$ events are observed.

- Good spatial resolution and timing information can help reject background processes.

- The nuclear theory is better understood in some isotopes than others. The interpretation of limits or signals might be easier to interpret for some isotopes.

No experiment, past or proposed, is able to optimize for all of these characteristics simultaneously. Each has chosen a design that emphasizes different aspects of this list. Similar points concerning an ideal experiment were made in Ref. 13 and also by Yu. Zdesenko in Ref 17 .

\section{Past Experiments}

The best experiments to date are the Ge experiments. The IGEX experiment 18 and the Heidelberg-Moscow experiment $[19$ have produced the most restrictive limits on the half-life and deduced $\left\langle m_{\beta \beta}\right\rangle$. The results from these two experiments along with many others are given in Table 1 From the Ge experiments the limit on $\left\langle m_{\beta \beta}\right\rangle$ is about 300 to $1300 \mathrm{meV}$, depending on the chosen matrix elements.

One intriguing bit of history helps motivate the need for more than one $\beta \beta(0 \nu)$ effort. An early spectrum in a Te experiment ${ }^{28}$ saw a peak at the $2.53-\mathrm{MeV}$ endpoint with $\approx 2 \sigma$ confidence. At a similar point in time, a Ge spectrum 29 showed a peak of unknown origin at the same energy. If the Ge peak was real, it would mean that a dangerous unidentified $\gamma$ ray existed that would be a background for any ${ }^{130} \mathrm{Te}$ experiment. However, both peaks turned out to be spurious. The Te peak faded with additional statistics and the Ge peak was an electronic artifact. The moral of the story is that a peak, by itself, may not suffice to prove an observation of $\beta \beta(0 \nu)$. But two peaks in different isotopes from experiments using different techniques would be convincing. This would be especially true if the rates were consistent with a common $\left\langle m_{\beta \beta}\right\rangle$.

A recent claim for $\beta \beta(0 \nu)$ was made by a small subset of the Heidelberg- 
Table 1. Best reported limits on $T_{1 / 2}^{0 \nu}$. The $\left\langle m_{\nu}\right\rangle$ limits and ranges are those deduced by the authors and their choices of matrix elements within the cited experimental papers. All are quoted at the $90 \%$ confidence level except as noted.

\begin{tabular}{|c|c|c|c|}
\hline Isotope & $\mathrm{T}_{1 / 2}^{0 \nu}(\mathrm{y})$ & $\left\langle m_{\beta \beta}\right\rangle(\mathrm{eV})$ & Reference \\
\hline${ }^{48} \mathrm{Ca}$ & $>9.5 \times 10^{21}(76 \%)$ & $<8.3$ & 20 \\
\hline \multirow[t]{2}{*}{${ }^{76} \mathrm{Ge}$} & $>1.9 \times 10^{25}$ & $<0.35$ & 19 \\
\hline & $>1.6 \times 10^{25}$ & $<0.33-1.35$ & 18 \\
\hline${ }^{82} \mathrm{Se}$ & $>2.7 \times 10^{22}(68 \%)$ & $<5$ & 21 \\
\hline${ }^{100} \mathrm{Mo}$ & $>5.5 \times 10^{22}$ & $<2.1$ & 22 \\
\hline${ }^{116} \mathrm{Cd}$ & $>7 \times 10^{22}$ & $<2.6$ & 23 \\
\hline${ }^{128,130} \mathrm{Te}$ & $\begin{array}{l}\frac{T_{1 / 2}(130)}{T_{1 / 2}(128)}=(3.52 \pm 0.11) \times 10^{-4} \\
\text { (geochemical) }\end{array}$ & $<1.1-1.5$ & 24 \\
\hline${ }^{128} \mathrm{Te}$ & $>7.7 \times 10^{24}$ & $<1.1-1.5$ & 24 \\
\hline${ }^{130} \mathrm{Te}$ & $>1.4 \times 10^{23}$ & $<1.1-2.6$ & 25 \\
\hline${ }^{136} \mathrm{Xe}$ & $>4.4 \times 10^{23}$ & $<1.8-5.2$ & 26 \\
\hline${ }^{150} \mathrm{Nd}$ & $>1.2 \times 10^{21}$ & $<3$ & 27 \\
\hline
\end{tabular}

Moscow collaboration 3031. This claim has generated a fair amount of controversy 3233343536 . The analysis in Ref. 31 chooses a background model that contains many peaks in an extended region and therefore limits its analysis to a narrow region about the $\beta \beta(0 \nu)$ peak. This analysis finds a probability that a peak exists at the $\beta \beta(0 \nu)$ location is $96.5 \%$ or $2.1 \sigma$. A wide-region analysis assuming that only a continuous background is present, finds a probability of only $\approx 65 \%$ that a peak exists. These two extremes in the background model indicate that the significance of the result is sensitive to the background description. The quoted significance of the result $(2.1 \sigma)$, however, does not include a systematic uncertainty related to the choice of background model. If it did, the significance would decrease. Taken at face value, a $2.1 \sigma$ result may be intriguing, but it certainly does not warrant a strong claim for evidence.

\section{The Various Proposals}

The various proposals are too numerous to describe all in detail. I have listed all the proposals with which I am familiar in Table 2 To give the reader a feel for the proposals, I have chosen 5 to profile: CUORE, EXO, GENIUS, Majorana, and MOON. The important point to take away from Table 2 is that many of the proposals claim a sensitivity to the desired target of $50 \mathrm{meV}$ (i.e. $\approx 10^{27} \mathrm{y}$ ). For many of the proposals described in Table 2 the previous experiments listed in Table 1 are effective prototypes. This fact helps build confidence that the next generation of experiments will reach their intended goal. 
Table 2. A summary of the double-beta decay proposals. The quoted sensitivities are those quoted by the proposers but scaled for 5 years of run time. These sensitivities should be used carefully as they depend on background estimates for experiments that don't exist yet.

\begin{tabular}{|c|c|c|c|}
\hline Experiment & Source & Detector Description & $\begin{array}{l}\text { Sensitivity to } \\
T_{1 / 2}^{0 \nu}(\mathrm{y})\end{array}$ \\
\hline COBRA 37 & ${ }^{130} \mathrm{Te}$ & $10 \mathrm{~kg} \mathrm{CdTe} \mathrm{semiconductors}$ & $1 \times 10^{24}$ \\
\hline $\mathrm{DCBA}^{38}$ & ${ }^{150} \mathrm{Nd}$ & $20 \mathrm{~kg}{ }^{e n r} \mathrm{Nd}$ layers between tracking chambers & $2 \times 10^{25}$ \\
\hline NEMO 39 & ${ }^{100} \mathrm{Mo}$ & $10 \mathrm{~kg}$ of $\beta \beta(0 \nu)$ isotope $(7 \mathrm{~kg} \mathrm{Mo})$ with tracking & $4 \times 10^{24}$ \\
\hline CAMEO 40 & ${ }^{116} \mathrm{Cd}$ & $1 \mathrm{t} \mathrm{CdWO}_{4}$ crystals in liq. scint. & $1 \times 10^{27}$ \\
\hline CANDLES41 & ${ }^{48} \mathrm{Ca}$ & several tons of $\mathrm{CaF}_{2}$ crystals in liq. scint. & $1 \times 10^{26}$ \\
\hline CUORE 42 & ${ }^{130} \mathrm{Te}$ & $750 \mathrm{~kg} \mathrm{TeO}_{2}$ bolometers & $2 \times 10^{26}$ \\
\hline $\mathrm{EXC} 43$ & ${ }^{136} \mathrm{Xe}$ & $1 \mathrm{t}{ }^{e n r} \mathrm{Xe}$ TPC (gas or liquid) & $8 \times 10^{26}$ \\
\hline GEM 44 & ${ }^{76} \mathrm{Ge}$ & $1 \mathrm{t}{ }^{e n r} \mathrm{Ge}$ diodes in liq. nitrogen & $7 \times 10^{27}$ \\
\hline GENIUS45 & ${ }^{76} \mathrm{Ge}$ & $1 \mathrm{t} 86 \%{ }^{\text {enr }} \mathrm{Ge}$ diodes in liq. nitrogen & $1 \times 10^{28}$ \\
\hline GSO 4647 & ${ }^{160} \mathrm{Gd}$ & $2 \mathrm{t} \mathrm{Gd}_{2} \mathrm{SiO}_{5}$ : Ce crystal scint. in liq. scint. & $2 \times 10^{26}$ \\
\hline Majorand 48 & ${ }^{76} \mathrm{Ge}$ & 0.5 t $86 \%$ segmented ${ }^{e n r} \mathrm{Ge}$ diodes & $3 \times 10^{27}$ \\
\hline MOON 49 & ${ }^{100} \mathrm{Mo}$ & $34 \mathrm{t}^{\text {nat }}$ Mo sheets between plastic scint. & $1 \times 10^{27}$ \\
\hline $\mathrm{Xe}$ & ${ }^{136} \mathrm{Xe}$ & $1.56 \mathrm{t}$ of ${ }^{e n r} \mathrm{Xe}$ in liq. scint. & $5 \times 10^{26}$ \\
\hline XMASS 51 & ${ }^{136} \mathrm{Xe}$ & $10 \mathrm{t}$ of liq. $\mathrm{Xe}$ & $3 \times 10^{26}$ \\
\hline
\end{tabular}

\subsection{CUORE}

The success of the MIBETA experiment ${ }^{25}$ has resulted in the CUORE (Cryogenic Underground Observatory for Rare Events) proposa 52 . One thousand $\mathrm{TeO}_{2}$ crystals of $750 \mathrm{~g}$ each would be operated as a collection of bolometers. The detectors will be collected into 25 separate towers of 40 crystals. Each tower will have 10 planes of 4 crystals each. One such plane has already been successfully tested and a single tower prototype referred to as CUORICINO is scheduled to begin operation in spring 2003.

The energy resolution at the $\beta \beta(0 \nu)$ peak $(2.529 \mathrm{MeV})$ is expected to be about $5 \mathrm{keV}$ FWHM $(\approx 0.2 \%)$. The background has been measured in the first plane to be $\approx 0.5$ counts $/(\mathrm{keV} \cdot \mathrm{kg} \cdot \mathrm{y})$. However a major component of this background was due to a surface contamination arising from the use of cerium oxide polishing compound which tends to be high in thorium. With this problem solved, the experimenters project a conservative estimate of the background to be $\approx 0.01$ counts $/(\mathrm{keV} \cdot \mathrm{kg} \cdot \mathrm{y})$.

A major advantage of this proposal is that the natural abundance of ${ }^{130} \mathrm{Te}$ is $34 \%$ and, thus, no enrichment is needed resulting in significant cost savings. As with MIBETA, the cosmogenic activities within the $\mathrm{TeO}_{2}$ crystals are not a serious concern. On the other hand, the crystal mounts and cryostat form a significant amount of material close to the bolometers. Much of the cryostat is shielded with Roman period lead but a fair quantity of copper and Teflon remain close to the crystals. 


\section{2. $E X O$}

The Enriched Xenon Observatory (EXO 43 proposes to use up to $10 \mathrm{t}$ of $60-80 \%$ enriched ${ }^{136} \mathrm{Xe}$. The unique aspect of this proposal is the plan to detect the ${ }^{136} \mathrm{Ba}$ daughter ion correlated with the decay. If the technique is perfected, it would eliminate all background except that associated with $\beta \beta(2 \nu)$. The real-time optical detection of the daughter $\mathrm{Ba}$ ion, initially suggested in Ref. [53] might be possible if the ion can be localized and probed with lasers. The spectroscopy has been used for $\mathrm{Ba}^{+}$ions in atom traps. However, the additional technology to detect single $\mathrm{Ba}$ ions in a condensed medium or to extract single $\mathrm{Ba}$ ions from a condensed medium and trap them must be demonstrated for this application. To optically detect the alkali-like $\mathrm{Ba}^{+}$ion, it is excited from a $6^{2} \mathrm{~S}_{1 / 2}$ ground state to a $6^{2} \mathrm{P}_{1 / 2}$ with a 493 $\mathrm{nm}$ laser. Since this excited state has a $30 \%$ branching ratio to a $5^{4} \mathrm{D}_{3 / 2}$ metastable state, the ion is detected by re-exciting this metastable state to the $6 \mathrm{P}$ state via a $650-\mathrm{nm}$ laser and then observing the resulting decay back to the ground state. This procedure can be repeated millions of times per second on a single ion and produce a significant signal.

The EXO plan is to use Liquid Xe (LXe) scintillator. The LXe concept has the advantage of being much smaller than a gaseous TPC due to the high density of LXe. However, the higher density makes the scattering of the laser light too great to optically detect the $\mathrm{Ba}^{+}$in-situ. However, once the $\mathrm{Ba}$ ion is localized via its scintillation and ionization, it might be extracted via a cold finger electrode coated in frozen Xe (M. Vient, unpublished observation, 1991). The ion is electrostatically attracted to the cold finger which later can be heated to evaporate the Xe and release the $\mathrm{Ba}$ ion into a radio frequency quadrupole trap. At that point, the $\mathrm{Ba}^{++}$ is neutralized to $\mathrm{Ba}^{+}$, laser cooled and optically detected. The efficiency of the tagging has yet to be demonstrated and is a focus of current research.

The collaboration has recently performed experiments to optimize the energy resolution. By measuring both scintillation light and ionization simultaneously, they have achieved energy resolution sufficient for the experiment. Tests to determine the viability of the Ba extraction process are also being performed. The EXO collaboration has received funding to proceed with a 200-kg enriched Xe detector without Ba tagging. This initial prototype will operate at the Waste Isolation Pilot Plant (WIPP) in southern New Mexico.

\subsection{GENIUS}

The progress and understanding of Ge detectors has been developed over more than 30 years of experience. The potential of these detectors lie in their great energy resolution, ease of operation, and the extensive body of experience relating to the reduction of backgrounds. This potential is not yet exhausted as is evidenced by the GENIUS and Majorana proposals that build on the experimenters' previous efforts.

The GENIUS (GErmanium NItrogen Underground Setup) ${ }^{45}$ proposal has 
evolved from the Heidelberg-Moscow (HM) experiment. The driving design principle behind this proposed Ge detector array experiment is the evidence that the dominant background in the HM experiment was due to radioactivity external to the Ge. (The reader should contrast this with the motivation for the design of the Majorana proposal described below.) An array of 2.5-kg, p-type Ge crystals would be operated "naked" within a large liquid nitrogen (LN) bath. By using naked crystals, the external activity would be moved to outside the LN region. P-type crystals have a dead layer on the external surface that reduces their sensitivity to external $\beta$ and $\alpha$ activity. Due to its low stopping power, roughly $12 \mathrm{~m}$ of LN is required to shield the crystals from the ambient $\gamma$-ray flux at the intended experimental site at Gran Sasso. By immersion in LN, the optimal operating temperature is maintained without a bulky cryostat and a test of the naked operation of a crystal in a 501 dewar has been successful $[5455]$. The results from a several-week operation indicate that the performance of the detector was comparable to those operated in a conventional vacuum-tight cryostat system. Their measurements also indicate very little cross talk between naked detectors and that an extended distance $(\approx 6$ $\mathrm{m})$ between the FET and the crystal does not degrade the energy resolution.

The proposal anticipates an energy resolution of $\approx 6 \mathrm{keV} \mathrm{FWHM}(0.3 \%)$ and a threshold of $11 \mathrm{keV}$. The value of this low threshold is set by $\mathrm{x}$ rays from cosmogenic activities. Using $1 \mathrm{t}$ of $86 \%$ enriched Ge detectors, the target mass is large enough for dark matter studies. In fact a $40-\mathrm{kg}{ }^{n a t} \mathrm{Ge}$ proof-of-principle experiment has been approved for dark matter studies.

\subsection{Majorana}

The Majorana proposa 18 (named in honor of Etorre Majorana) involves many of the IGEX collaborators. Their analysis indicated that ${ }^{68} \mathrm{Ge}$ contained within the Ge detectors was the limiting background for their $\beta \beta(0 \nu)$ search. (Contrast this with the GENIUS approach described above.) The proposal's design therefore emphasizes segmentation and pulse shape discrimination to reject this background. The electron capture of ${ }^{68} \mathrm{Ge}$ is not a significant problem but ${ }^{68} \mathrm{Ge}$ decays to the $\beta^{+}$emitting ${ }^{68} \mathrm{Ga}$. This isotope can create background in the $\beta \beta(0 \nu)$ window if one of the annihilation $\gamma$ rays converts within the crystal. The energy deposits of the positron and $\gamma$ ray may pollute the peak window in energy, but the deposits will be separated in space. In contrast, a $\beta \beta(0 \nu)$ event will have a localized energy deposit. Segmentation of the crystals permits a veto of such events. Furthermore, distinct ionization events will have a different pulse shape than a localized event. Therefore pulse shape analysis can also help reject background. Majorana plans to use $\approx 500$, $86 \%$ enriched, segmented Ge crystals for a total of $500 \mathrm{~kg}$ of detector. The cryostat would be formed from very pure electroformed $\mathrm{Cu}[56$. 


\subsection{MOON}

The MOON (Mo Observatory Of Neutrinos) proposa $\frac{49}{49}$ plans to use ${ }^{100}$ Mo as a $\beta \beta(0 \nu)$ source and as a target for solar neutrinos. This dual purpose and a sensitivity to low-energy supernova electron neutrinos $\frac{57}{}$ make it an enticing idea. ${ }^{100}$ Mo has a high Q-value $(3.034 \mathrm{MeV})$, which results in a large phase space factor and places the $\beta \beta(0 \nu)$ peak region well above most radioactive backgrounds. It also has hints of a favorable $\left|M_{0 \nu}\right|$ but unfortunately it has a fast $T_{1 / 2}^{2 \nu}$. The experiment will make energy and angular correlation studies of $\beta \beta$ to select $\beta \beta(0 \nu)$ events and to reject backgrounds. The planned MOON configuration is a supermodule of scintillator and Mo ensembles. One option is a module of plastic fiber scintillators with thin $(0.03$ $\mathrm{g} / \mathrm{cm}^{2}$ ) layers of claded Mo, which are arranged to achieve a position resolution comparable to the fiber diameter $(2-3 \mathrm{~mm})$. A total of 34 tons of natural Mo would be required.

As a solar neutrino detector, ${ }^{100} \mathrm{Mo}$ has a low threshold: $168 \mathrm{keV}$, and the estimated observed event rate is $\approx 160 /$ (ton ${ }^{100}$ Mo.year) without neutrino oscillations. It is sobering to realize that the primary background for the delayed-coincidence solar neutrino signal is accidental coincidences between $\beta \beta(2 \nu)$ decays.

The project needs Mo and scintillator radioactive impurity levels of better than $1 \mathrm{mBq} /$ ton. This can be achieved by carbonyl chemistry for Mo and plastics can be produced cleanly. However, the total surface area of the Mo-scintillator modules is $\approx 26000 \mathrm{~m}^{2}$. Dust, being electrostatically charged, tends to garner Rn daughters and becomes radioactive. Keeping these surfaces clean of dust during production and assembly will be a challenge. Liquid scintillator and bolometer options that would avoid this large surface area are also being considered. The simulations of the design indicate that the energy resolution for the $\beta \beta(0 \nu)$ peak will be $\approx 7 \%$ which is at the upper end of the range of feasibility for a sub $50 \mathrm{meV}\left\langle m_{\nu}\right\rangle$ experiment. The bolometer option would also remove the resolution concerns. Use of enriched ${ }^{100} \mathrm{Mo}$ is feasible, and would reduce the total volume of the detector and source ensemble resulting in a lower internal radioactivity contribution to the background by an order of magnitude.

\subsection{OTHER PROPOSALS}

There are too many proposals for detailed description so I have summarized those of which I am aware in Table 2 and mention them here. The CAMEO proposa 40 would use $1000 \mathrm{~kg}$ of scintillating ${ }^{116} \mathrm{CdWO}_{4}$ crystals situated within the Borexino apparatus. The Borexino liquid scintillator would provide shielding from external radioactivity and light piping of crystal events to the photomultiplier tube (PMT) array surrounding the system. Early phases of the program would use the Borexino counting test facility. Similarly, the CANDLES proposa 41 (CAlcium floride for study of Neutrino and Dark matter by Low Energy Spectrometer) plans to immerse $\mathrm{CaF}_{2}$ in liquid scintillator. The scintillation light from the $\beta \beta$ of ${ }^{48} \mathrm{Ca}$ will be detected via PMTs. The low isotopic abundance $(0.187 \%)$ of ${ }^{48} \mathrm{Ca}$ requires a very 
large operating mass. Two groups $\frac{4647}{4}$ have been studying the use of GSO crystals $\left(\mathrm{Gd}_{2} \mathrm{SiO}_{5}\right.$ :Ce) for the study of ${ }^{160} \mathrm{Gd}$.

COBRA (CdTe O neutrino double Beta Research Apparatus) ${ }^{37}$ would use CdTe or $\mathrm{CdZnTe}$ semiconductors to search for $\beta \beta(0 \nu)$ in either $\mathrm{Cd}$ or Te. $16001-\mathrm{cm}^{3}$ crystals would provide $10 \mathrm{~kg}$ of material. GEM is a proposa 44 that is very similar to that of GENIUS. However, much of the LN shielding would be replaced with high purity water.

The Drift Chamber Beta-ray Analyzer (DCBA) proposa $\sqrt{38}$ is for a threedimensional tracking chamber in a uniform magnetic field. A drift chamber inside a solenoid and cosmic-ray veto counters comprises the detector. Thin plates of $\mathrm{Nd}$ would form the source. The series of NEMO experiments is progressing with NEMO339 beginning operation in 2002. In concept the detector is similar to NEMO-2. That is, it contains a source foil enclosed between tracking chambers that is itself enclosed within a scintillator array. NEMO-3 can contain a total of $10 \mathrm{~kg}$ of source and plans to operate with several different isotopes, but with ${ }^{100}$ Mo being the most massive at $7 \mathrm{~kg}$. The collaboration is also discussing the possibility of building a 100-kg experiment that would be called NEMO-4.

There are two additional groups proposing to use ${ }^{136} \mathrm{Xe}$ to study $\beta \beta(0 \nu)$. Caccianiga and Giammarch: 150 propose to dissolve $1.56 \mathrm{t}$ of enriched Xe in liquid scintillator. The XMASS 51 collaboration proposes to use $10 \mathrm{t}$ of liquid xenon for solar neutrino studies. The detector would have sensitivity to $\beta \beta(0 \nu)$.

\section{The Matrix Elements}

In order to determine an effective Majorana neutrino mass from a $\beta \beta(0 \nu)$ result, one requires input from nuclear theory. The matrix elements however are very hard to calculate and the various techniques and authors produce varying results. Reference 58 includes a rather complete list of references and the results of calculation of the matrix elements. The two techniques for calculating the matrix elements are the quasiparticle random phase approximation (QRPA) and the nuclear shell model. Each approach has its advantages and disadvantages and hence critics and advocates. Due to this complicated situation, the spread in the calculations is commonly taken as a measure of the theoretical uncertainty.

For a given $\left\langle m_{\beta \beta}\right\rangle$, the spread in predicted half-lives is approximately an order of magnitude. Thus the spread in $\left\langle m_{\beta \beta}\right\rangle$ deduced from a potential $\beta \beta(0 \nu)$ measurement is a factor of 3 . It should be emphasized that the qualitative physics conclusions arising from an observation of $\beta \beta(0 \nu)$ are so profound, that this factor of 3 is a detail. However, precise interpretation of any result will be limited due to this spread. The spread is due to the community's choice to include all calculations when estimating the theoretical uncertainty. No consensus has been reached identifying the calculations that are outdated or relatively less precise. Naively, one concludes that the factor of 3 is an overestimate. At present this uncertainty is large enough that potential conclusions regarding the mass spectrum 13 or the Ma- 
jorana phases 59 might be diminished. Improvement in the calculations will have a big payoff if $\beta \beta(0 \nu)$ is observed.

More important, however, is the need for confirmation as described above. If observations of $\beta \beta(0 \nu)$ are made in two isotopes, a comparison between the two deduced $\left\langle m_{\beta \beta}\right\rangle$ values might indicate that they are consistent. Obviously this would be strong evidence that $\beta \beta(0 \nu)$ is truly the correct interpretation. For this argument to be persuasive, however, confidence in the theory and its estimated uncertainties must be sufficient that critics concede the two $\left\langle m_{\beta \beta}\right\rangle$ values are consistent.

Encouragingly, the technology available for such calculations has improved, especially the shell model techniques. The physics motivation for these experiments is very strong, so perhaps interest in the theoretical aspect of this problem will build also.

\section{Conclusions}

It is a very exciting time for $\beta \beta(0 \nu)$. The next generation of experiments will have a sensitivity of about $50 \mathrm{meV}$ for $\left\langle m_{\beta \beta}\right\rangle$ : the critical target of $\sqrt{\delta m_{\text {atm }}^{2}}$. Therefore future experiments will have a known neutrino mass with which to compare. As a result, even null experiments will impact the question of whether neutrinos are Dirac or Majorana in certain mass-spectrum configurations.

\section{Acknowledgments}

After the announcement of the 2002 Nobel Prize for physics, this conference evolved into a tribute to Ray Davis and his great achievements that culminated with his winning this prestigious award. I wish to join the numerous scientists to congratulate him on a spectacular career. I had the pleasure to collaborate with Ray on the Russian-American Gallium solar neutrino Experiment (SAGE) and, therefore, I also wish to thank him personally for his inspiring example.

I wish to thank Frank Avignone and Peter Doe for critical readings of this manuscript. This research was sponsored in part by DOE grant W-7405-ENG-36.

\section{References}

1. T. Kajita and Y. Totsuka, Rev. Mod. Phys. 73,85 (2001).

2. Y. Fukuda, et al., Phys. Lett. B 335, 237 (1994) .

3. R.A. Becker-Szendy, et al., Phys. Rev. D46, 3720 (1992).

4. W.W.M. Allison, et al., Phys. Lett. B 449, 137 (1999).

5. B.T. Cleveland, et al., Astrophys. J. 496, 505 (1998).

6. J.N. Abdurashitov, et al., Phys. Rev. C60, 055801 (1999).

7. W. Hampel, et al., Phys. Lett. B447, 127 (1999).

8. Q.R. Ahmad, et al., Phys. Rev. Lett. 87, 071301 (2001); ibid 89, 001301 (2002); ibid 89, 001302 (2002).

9. S. Fukuda, et al., Phys. Rev. Lett. 86, 5651 (2001); ibid 86, 5656 (2001).

10. M. Apollonio, et al., Phys. Lett. B466, 415 (1999). 
11. F. Boehm, et al., Phys. Rev. D64, 112001 (2001).

12. K. Eguchi, et al., hep-ex/0212021

13. Steven R. Elliott, and Petr Vogel, Annu. rev. Nucl. Part. Sci. in press.

14. A. Osipowicz, et al. hep-ex/0109033

15. A. Aguilar, et al., Phys. Rev. D64, 112007 (2001).

16. Elliott SR, Hahn AA, and Moe MK, Phys. Rev. Lett. 59, 2020 (1987).

17. Yu. Zdesenko, J. Phys. G: Nucl. Part. Phys. 27, 2129 (2001); Rev. Modern Phys., 74, 663 (2002); Phys. Atom. Nucl. 65, 1 (2002); Nucl. Phys. B, 110, 385 (2002).

18. C.E. Aalseth, et al., Phys. Rev. C59, 2108 (1999); Nucl. Phys. of the Russian Academy of Science 63, 1299 (2000); hep-ex/0202026 (2002).

19. H.V. Klapdor-Kleingrothaus, et al., Eur. Phys. J. A12:147 (2001)

20. You Ke, et al., Phys. Lett. B265, 53 (1991).

21. S.R. Elliott, et al., Phys. Rev. C46:1535 (1992)

22. H. Ejiri, et al., Phys. Rev. C63, 065501 (2001).

23. F.A. Danevich, et al., Phys. Rev. C62:044501 (2000)

24. T. Bernatowicz, et al., Phys. Rev. C47:806 (1993)

25. A. Alessandrello, et al., Phys. Lett. B486, 13 (2000).

26. R. Luescher, et al., Phys. Lett. B434, 407 (1998).

27. A. De Silva, et al., Phys. Rev. C56:2451 (1997)

28. Nucl. Phys. (Proc. Supp.) B35, 366 (1994).

29. D. Reusser, et al., Phys. Rev. D45, 2548 (1992).

30. H.V. Klapdor-Kleingrothaus, et al., Mod. Phys. Lett. 16, 2409 (2001).

31. H.V. Klapdor-Kleingrothaus, Foundations of Physics 32, 1181 (2002).

32. C.E. Aalseth, et al., Mod. Phys. Lett. 17, 1475 (2002);

33. H.V. Klapdor-Kleingrothaus, hep-ph/0205228 (2002);

34. H.L. Harney, hep-ph/0205293 (2002);

35. F. Feruglio, A. Strumia, and F. Vassani, Nucl.Phys. B637, 345 (2002).

36. Yu.G. Zdesenko, F.A. Danevich, and V.I. Tretyak, Phys. Lett. B546, 206 (2002).

37. K. Zuber, Phys.Lett. B519, 1 (2001).

38. N. Ishihara, et al., Nucl. Instrum. Meth. A443, 101 (2000).

39. X. Sarazin, et al., hep-ex/0006031

40. G. Bellini, et al., Eur. Phys. J. C19, 43 (2001).

41. T. Kishimoto, et al., Osaka University Laboratory for Nuclear Studies Annual Report.

42. A. Alessandrello, et al., hep-ex/0201038

43. M. Danilov, et al., Phys. Lett. B480, 12 (2000).

44. Yu.G. Zdesenko, O.A. Ponkratenko, and V.I. Tretyak, J. Phys. G27, 2129 (2001).

45. H.V. Klapdor-Kleingrothaus, hep-ph/0103074

46. F.A. Danevich, et al., Nucl.Phys. A694,375 (2001) .

47. S.C. Wang, H.T. Wong, and M. Fujiwara, hep-ex/0009014 submitted to Astropart. Phys..

48. C.E. Aalseth, et al., hep-ex/0201021

49. H. Ejiri, et al. Phys. Rev. Lett. 85, 2917 (2000).

50. B. Caccianiga, and M.G. Giammarchi, Astropart. Phys. 14, 15 (2001).

51. S. Moriyama, et al., Presented at XENON01 workshop, December 2001, Tokyo, Japan.

52. S. Pirro, et al., Nucl. Instrum. Meth. A444, 71 (2000).

53. M.K. Moe, Phys. Rev. C44:R931 (1991)

54. H.V. Klapdor-Kleingrothaus, J. Hellmig, M. Hirsch, J. Phys. G24, 483 (1998).

55. L. Baudis, et al., Nucl. Instrum. Meth. A426, 425 (1999).

56. R.L. Brodzinski, et al., Nucl. Instrum. Meth. A292:337 (1990)

57. H. Ejiri, J. Engel, and N. Kudomi, astro-ph/0112379 (2001). 
5 June 2018 23:7 WSPC/INSTRUCTION FILE Elliott`BBDK

16 Steven R. Elliott

58. J. Suhonen and O. Civitarese, Phys. Rep. 300:123 (1998)

59. V. Barger, et al., Phys. Lett. B540, 247 (2002). 